\title{
Super-Absorbent Polymer: A Review on the Characteristics and Application
}

\author{
Isha Meshram, Vrushali Kanade, Nidhisha Nandanwar, Pradnya Ingle* \\ Department of Chemical Engineering, Shivajirao S. Jondhale College of Engineering, Dombivli (E), University \\ of Mumbai, India
}

*Corresponding Author: Pradnya Ingle, Associate Professor, Department of Chemical Engineering, Shivajirao S. Jondhale College of Engineering, Dombivli (E), University of Mumbai, India

\begin{abstract}
Super absorbency is superior swelling originates to absorb and retain large amount of aqueaous solution. Superabsorbent polymers are cross linked network of hydrophilic polymer chains. Poly (acrylate/). Poly (acrylate/acrylic acid) was synthesized to form superabsorbent hydrogel (SAH) and their performance as a water retaining aid for irrigation were assessed. The SAH acrylatelacrylic acid was cross-linked with $N N^{\prime}-$ methylene-bis-acrylamide for optimum water retention. With today's demand for a one click solution, the development of superabsorbent technology has been largely directed by the needs of disposable hygiene segment, agricultural industry etc. Over the years, starch-grafted superabsorbent polymer turned into crosslinked acrylic homopolymers. Finally, the concept, mechanism and major application areas of superabsorbent are discussed well.
\end{abstract}

Keywords: superabsorbent hydrogel, water retention

\section{INTRODUCTION}

Superabsorbent polymers (SAP) are a class of polymers that are able to absorb large amount of water, typically more than traditional absorbent material. Generally they consist of a network of polymer chain that are cross-linked to avoid dissolution. Usually that are ionic functional group along the polymer chain to facilitate diffusion of water within the network. Poly acrylate is the principal material used in SAP industry. Superabsorbent poly acrylates are prepared by polymerizing acrylic acid with a cross linker. When water comes into contact with one of these chains, it is drawn into the molecule by osmosis. Water rapidly migrates into the interior of the polymer network where it is stored. Type and degree of cross liners governability of the superabsorbent polymer to absorb and retain large volume of water and aqueous solution. These polymers have the ability to swell in water and retaining a significant fraction $(>20 \%)$ of water within their structure, without dissolving in water. Water uptake potential of these material goes as high as $100,000 \%$ of its own weight in a short period of time. The maximum volume of superabsorbent polymer covers the synthetic or petrochemical source and mostly acrylic acid and its sodium or potassium salts, and acrylamide issued in the industrial production of superabsorbent polymers. Nevertheless, superabsorbent polymers produced from proteins and polysaccharides like chitin, cellulose starch, and natural gums, are the green alternatives. Hydrogels that are responsive to specific molecules, such as glucose or antigens can be used as biosensors as well as drug delivery system (DDS). These kinds of hydrogels are also used as controlled- release delivery devices for bio active agents and agrochemicals. Contact lenses are also based on hydrogels. Special hydrogels are superabsorbent material are widely employed in hygienic used particularly disposable diaper and female napkins where they can capture secreted fluids, e.g., urine, blood, etc. agriculture grade of such hydrogels are used as granules for holding soil moisture in arid areas.[3]

\section{Materials AND Methods}

\subsection{Absorbing Versus Super Absorbing Materials}

The hygroscopic material usually categorized into two main class based on the major mechanism of water absorption, i.e., chemical and physical absorptions. Chemical absorbers (e.g., metal hydrides) catch water via chemical reaction converting their nature. Physical absorbers imbibe water via four main mechanism; reversible changes of their crystal structure(e.g., silica gel and anhydrous inorganic 
salts); physical entrapment of water via capillary forces in their macro-porous structures(e.g., soft polyurethane sponge); a combination of the mechanism (ii) and hydration of functional group (e.g., tissues paper); the mechanism which maybe anticipated by combination of mechanism of (ii) and (iii) and essentially dissolution and thermodynamically favored expansion of the macro- molecular chain limited by cross-linkages. Superabsorbent polymer (SAP) material fit in the latter category, yet they are organic material enormous capability of water absorption. SAPs as hydrogel, relative to their own mass can absorb and retain extraordinary large amounts of water or aqueous solution these ultra-high absorbing materials can imbibe deionized water as high as 1,000-100,000\% (10-1000 g/g) whereas the absorption capacity of common hydrogels is not more than $100 \%(1 \mathrm{~g} / \mathrm{g})$.[13] Visual and schematic illustrations of an acrylic- based anionic superabsorbent hydrogel in the dry and water swollen states are given in fig. 1

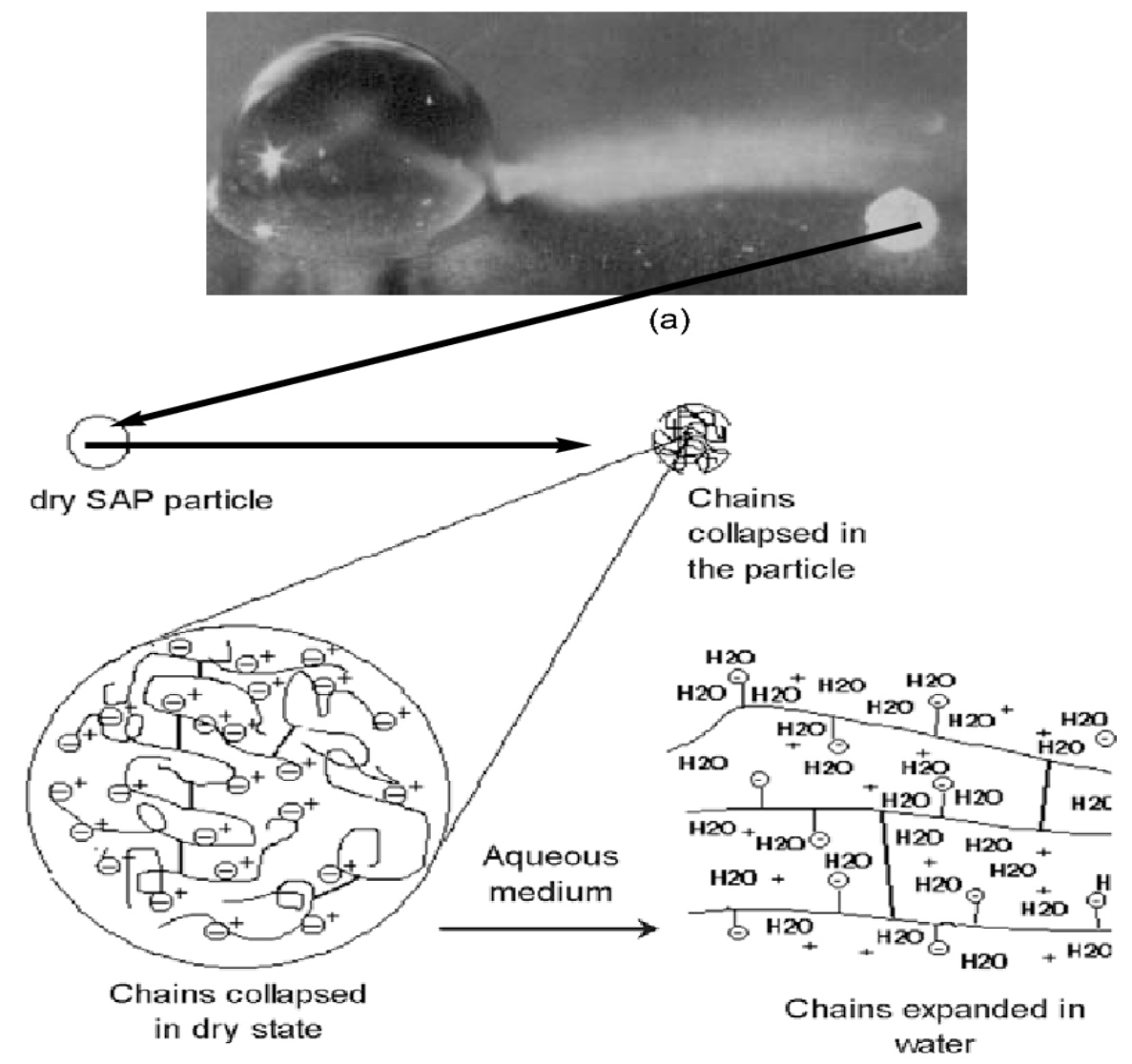

(b)

Figure1. Illustration of a typical acrylic-based anionic SAP material: (a) A visual comparison of the SAP single particle in dry and swollen state. The sample is a bead prepared from the inverse-suspension polymerization technique. (b)

Table1. Water absorbency of some common absorbent material in comparison with a typical commercial SAP sample.

\begin{tabular}{|c|c|}
\hline Absorbent Material & Water Absorbency (wt. \%) \\
\hline Whatman No. 3 filter paper & 180 \\
\hline Facial tissue paper & 400 \\
\hline Soft polyurethane sponge & 1050 \\
\hline Wood pulp fluff & 1200 \\
\hline Cotton ball & 1890 \\
\hline Superb A-200 & 20200 \\
\hline
\end{tabular}

Commercial SAP is generally sugar like hydroscopic material with white-light yellow color. The SAP particle shape (granule, fiber, film, etc.) has to be basically preserved after water absorption and swelling, i.e., the swollen gel strength should be high enough to prevent a loosening, mushy, or slimy state. This is a major practical feature that dis-criminates SAPs from other hydrogel. Traditional absorbent material (such as tissues paper and polyurethane foams) unlike SAPs, will lost most of their absorbed water when they are squeezed. Table compares water absorptiveness of some common absorbent material with a typical sample of a commercially available SAP 


\subsection{Physical Properties of Monomers}

The monomers useful for making superabsorbent polymer are water soluble monomer such as Acrylic acid (AA), meth acrylic acid, and 2-acrylamide (DAA) 2-methylpropanesulfuric acid are the principal ionizable monomers useful for making superabsorbent polymers. Other co-monomers such as acrylamide and $\mathrm{N}$-isopropyl acrylamide impart temperature sensitivity into the superabsorbent polymer. The useful cross-linkers include a variety of multifunctional monomers. They can be di-, tri-, or tetra functional, and can have mixed type of polymerizable groups such as methacrylate and ally, as inn ally methacrylate. Mixed types of functional groups provide olefins with varying reactivity towards the main monomer. The mixed-functional cross-linkers can be used to control the incorporation of the cross-link during the polymerization. For example, the gel point can be delayed to higher conversion of monomer. Conversely, the gel point can be delayed to higher conversion of the monomer by using a less reactive monomer compare to main monomer.[5]

\subsection{Manufacturing of Monomer}

The principal monomer used in the manufacturing of superabsorbent polymer is acrylic acid. Acrylic acid is made by the oxidation of propene inn two step. First, propene is oxidized to acrolien, and then the acrolien is further oxidized to acrylic acid. Different mixed oxide catalyst are used for each step to optimize the yield and selectivity of the oxidation reaction. Technical-grade acrylic acid id isolated from the steam-quenched reaction gas by means of solvent extraction and distillation, and is used principally in the further preparation purified by distillation or by crystallization from the melt to afford the polymerization grade monomer.

\subsection{Types of SAPs}

Resembling the hydrogel family, the SAPs can also be classified based upon different aspects.

SAPs maybe categorized to four groups on basis of presence or absence of electrical charge located in the cross-linked chains.

- Non ionic

- Ionic (including anionic and cationic)

- Amphoteric electrolyte (ampholytic) containing both acidic and basic group

- Zwitterion (poly betaines) containing both anionic and cationic group in each structural repeating unit.

- For example, the majority of commercial SAP hydrogel are anionic. SAPs are also classified based on the type of monomeric unit used in their chemical structure, thus the most conventional SAPs are held in one of the following categories.

- Cross-linked poly acrylates and polyacrylamides

- Hydrolyzed cellulose-poly acrylonitrile (PAN) or starch-PAN graft copolymers

- Cross-linked copolymers of maleic anhydride

However, according to original sources, SAPs are often divided into two main classes; i.e., synthetic (petrochemical-based) and natural. The latter can be divided into two main groups, i.e., the hydrogels based on polysaccharides and other based on polypeptides (proteins). The natural SAPs are usually prepared through addition of some synthetic part onto the natural substrates, e.g., graft copolymerization of vinyl monomers on polysaccharides. It should be pointed out when the term "superabsorbent polymer" is used without specifying its type, it actually implies the most conventional type of SAPs, i.e., the anionic acrylic acid that comprises a copolymer network based on the partially neutralized acrylic acid (AA) or acrylamide (AM).[2,3,4]

\subsection{Synthetic SAPs}

The greatest volume of SAPs comprises full synthetic or of petrochemical origin. They are produced from the acrylic monomers, most frequently acrylic acid (AA), its salts and acrylamide (AM)

There are two general pathways to prepare acrylic acid SAP network, i.e., simultaneously polymerization and crosslinking by a polyvinyl cross-linker, and cross-linking of a pre polymer by a poly- functional cross-linker. More discussion on the synthetic SAPS are provided in the related sections.[3,4] 


\subsection{SAPs Properties Determination Factors}

SAP technical Features

- The functional features of an ideal SAP material can be listed as follows

- The highest absorption capacity (maximum equilibrium swelling) in saline.

- Desired rate of absorption depend upon the application requirement.

- The highest absorbency load (AUL).

- The lowest price.

- The lowest soluble content and residual monomer.

- Colorless, odourless, and absolute non toxicity.

- Photo stability.

- The highest durability and stability in the swelling environment and urging the storage.

- The highest biodegradability with formation of toxic species following the degradation.

- $\mathrm{pH}$ neutrality after swelling in water.

- Re-wetting capability (if required).

- Reaction variables

- Cross linker type and concentration.

- Initiator type and concentration.

- Monomer(s) type and concentration

- Type, size, and amount of inorganic particle incorporated (if any)

- Polymerization method.

- Polymerization temperature.

- Amount and type of the surfactant used.

- Stirrer/reactor geometry and rate of stirring.

- Porosity generating method or the amount and type of the porogen (if used).

- Drying; its temperature, method and time.

- Post treatment such as surface cross linking to enhance the swollen gel strength.

Each of the mention above variables has its own individual effects on the SAP properties
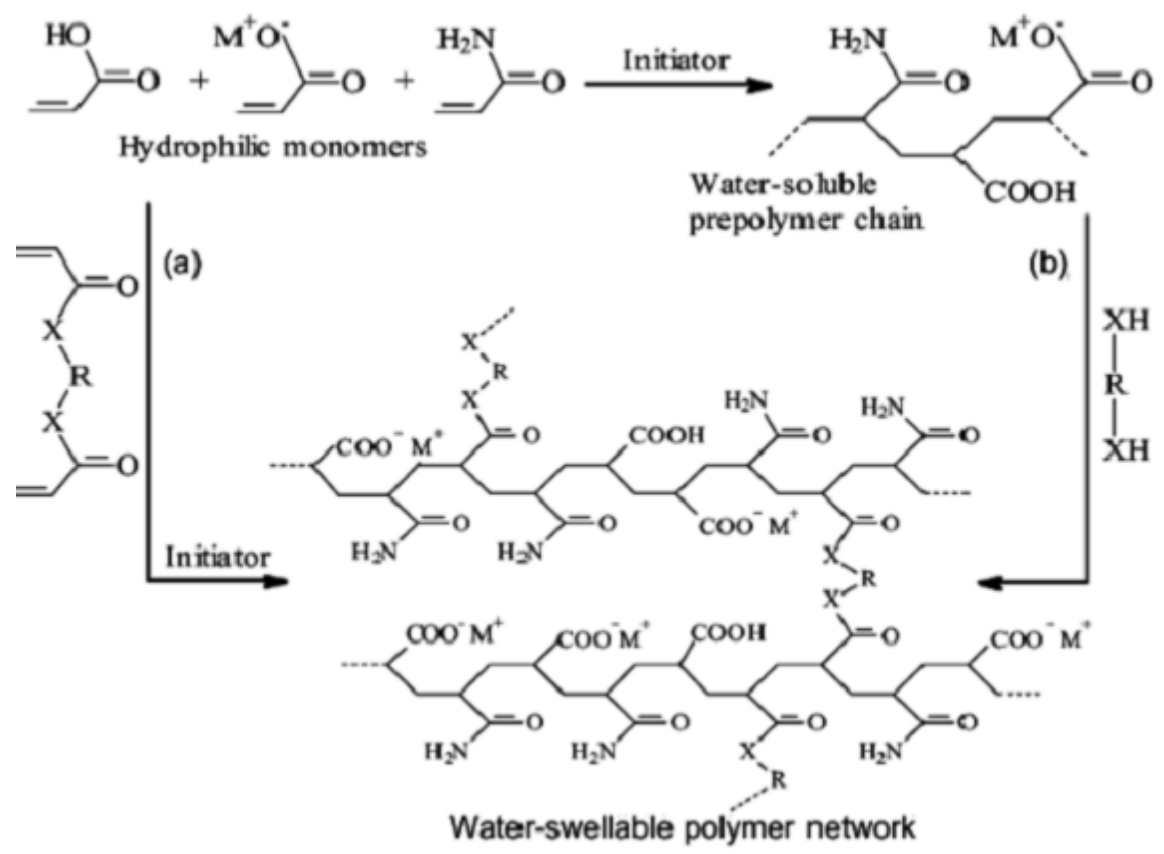

Figure2. Chemical structure of the reactants and general pathways to prepare an acrylic SAP network 
However, to optimize a process, a set of variables having the most special effects on the desired SAP product should be taken into consideration. [21]

\subsection{Synthetic Parameters}

Employing fixed type of reactants, the acrylic SAP properties are affected by the main synthetic factors abstracted. Many researchers have studied the effects of the preparative reaction variables on the SAP characteristics. These table contents have been actually extracted from numerous published. Additionally in recent years, researchers have partially focused on SAP composites and Nano composites to improve partially the mechanical and thermal properties of the hydrogels.

\subsection{Effect of Environmental Parameters on Properties}

The SAP particle physical specifications (e.g., size and porosity) as well as the swelling media also greatly affect their properties. These physical and environmental factors, particularly for acrylic anionic SAPs, have been studied widely by many researchers. Summarizes the results of plenty published works on the conventional SAPs properties.[5]

\subsection{Production Process: A Snap Shot}

Acrylic acid (AA) and its sodium or potassium salts, and acrylamide (AM) are most frequently used in the SAP industrial production. AM, a colorless liquid with vinegar odor, however, has a different story due to its ability to convert into its dimer (sub-section main starting materials). In the regard, the DAA level must be minimized to prevent the final product deficiencies, e.g., yield reduction, loss of soluble fraction, residual monomers, etc. Due to the potential problems originating from the inherent nature of AA to dimerize over time, manufacturers work properly with AA, such as timely order placement, justin-time delivery, moisture exclusion, and temperature-controlled storage (typically 17-18oC). In the laboratory scale syntheses, however, AA is often distilled before use, to purify and remove the impurities including the inhibitor and DAA. As salt solutions are usually produced by slow addition of appropriate solution of a desired metal hydroxide $(\mathrm{NaOH}$ or $\mathrm{KOH})$ to cooled AA while stirring mild. The temperature of this extremely exothermic neutralization reaction must be precisely controlled to prevent undersized polymerization. As mentioned before, the SAP materials are often synthesized through free-radically-initiated polymerization of acrylic monomers. The resins are prepared either in aqueous medium using solution polymerization or in a hydrocarbon medium where the monomers are well dispersed. These different methods are briefly discussed in the following sections. Some additional treatments, such as modified gel reduction, drying methods and grinding sieving, surface cross-liked and porosity generating techniques are important approaches for altering and fine-tuning the SAP morphology and physic-chemical properties.[5]

Solution Polymerization: - Free-radical initiated polymerization of AA and its salts (and AM), with a cross-linker is frequently used for SAP preparation. The carboxylic acid groups of the product are partially neutralized before or after the polymerization step. Initiation is most often carried out chemically with free radical ago or peroxide thermal dissociative species or by reaction of a reducing agent with an oxidizing agent (redox system). In addition, radiation is sometimes used for initiating the polymerization. The solution polymerization of AA and/or its salts with a water-soluble cross-linker, e.g., MBA in an aqueous solution is a straight forward process. The reactants are dissolved in water at desired concentration, usually about $10-70 \%$. A fast exothermic reaction yields a gel-like elastic product which is dried and the macro-porous mass is pulverized and sieve to obtain the required particle size. This preparative method usually suffers from the necessity to handle a rubbery/solid reaction product, lack of a sufficient reaction control, non-exact particle size distribution, and increasing the sol content mainly due to undesired effect of hydrolytic and thermal cleavage. However, for a general production of a SAP with acceptable swelling properties, the expensive and faster technique, i.e., solution method may often be preferred by the manufacture.[21]

\subsection{Inverse-Suspension Polymerization}

Dispersion polymerization is an advantageous method since the products are obtained as powder or microspheres, and thus grinding is not required. Since water-in-oil (W/O) process is chosen instead of the more common oil-in-water $(\mathrm{O} / \mathrm{W})$ the polymerization is referred as "inversion suspension". In this technique, the monomers and initiator are dispersed in the hydrocarbon phase as a homogenous mixture. The viscosity of the monomer solution, agitation speed, rotor design, and dispersant type mainly govern the resin particle size and shape. Some detailed discussion on hetrophase, polymerizations have already 
been published. The dispersion is thermodynamically unstable and require both continuous agitation and addition of a low hydrophilic-lipophilic-balance (HLB) suspending agent. The inverse suspension is a highly flexible and versatile technique to produce SAPs with high swelling ability and fast absorption kinetics. When the initiator dissolve in the dispersed phase, each particle contain all the reactive species and therefore behaves like an isolated micro-batch polymerization reactor. The resulting micro spherical particle are easily removed by filtration or centrifugation from the continuous organic phase. Upon drying, these particles or beads will directly provide a free flowing powder. In addition to the unique flowing properties of these beads, the inverse-suspension process display additional advantages compared to the solution method. These include a better control of the reaction heat removal, regulation of particle $=$-size distribution and further possibilities for adjusting.[3,4]

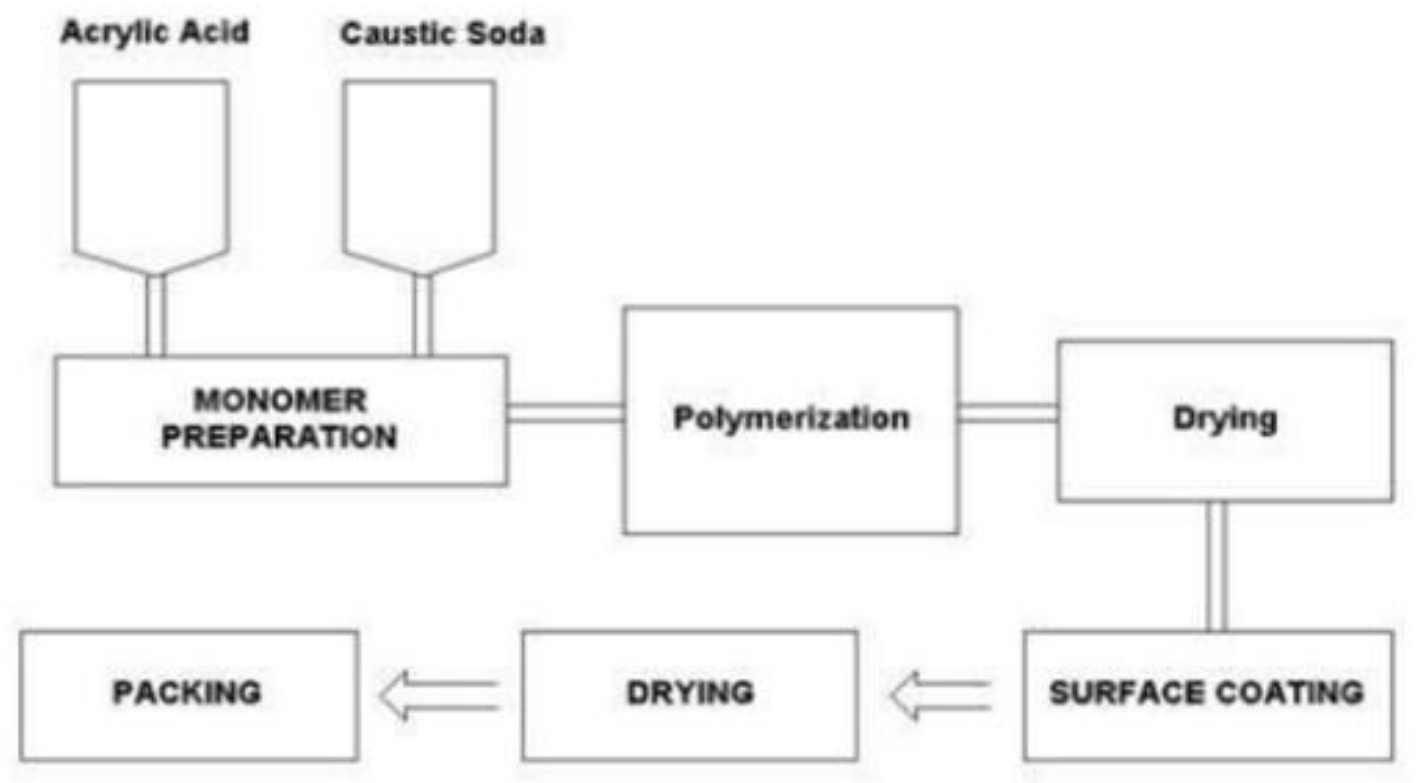

Figure3. Process flow diagram of super absorbent polymer

\section{Treatments ON POLYMERS}

\subsection{Gel Size Reduction}

After polymerization, the gel intermediate must be dried. However, prior to drying, the size of gel pieces must be reduced in order to increase surface area and speed drying. In some commercial process, the gel size reduction is done in the polymerization vessel by mean of high torque agitators or screws. In other processes, gel is sized in a separate unit operation of gel extrusion and cutting or mincing. Final gel particle are in the diameter range of $0.5-3 \mathrm{~cm}$.

\subsection{Drying}

The water used as solvent in the polymerization is removed from the polymerization is removed from the polymerized gel by evaporation in continuous-operation, hot air convection dryers or in contact dryer such as steam-heated drum dryers. In hot-air convection dryers, the rate of moisture removal depends on the heating gas temperature, humidity and flow rate, and the diffusion characteristic of waste from the gel. Drying occurs in three general stages. For high water content in the gel, the drying rate is constant, as the rate is limited by heat transfer into the gel. At low water contents, the drying is limited by diffusion of water through the gel, and the drying rate falls as water is further depleted. In the intermediate or transition regime, some of the polymer is still in the constant-rate regime while other portion have entered the diffusion-controlled regime. On rotating drum dryers, the drying energy is conductively transferred from the hot metal drum to gel. The hydrogel must be applied to the drum in a sufficiently thin layer, accounting for the available heat transfer and drum size, so that it is thoroughly dry in about three quarters of a revolution of the drum roll, where upon it is scraped from the drum with flaking knives. In order to spread the gel layer effectively onto the drum, the hydrogel must be soft enough to be deformable in the dryer nip. 


\subsection{Grinding and Sieving}

Grinding or milling the very coarse dryer product, followed by sieving sets the particle size distribution of the dried superabsorbent polymer. Two stage milling is frequently used in combination with sieving and recycles of the oversize particle in order to prepare a relatively narrow size distribution of the final granular powder. Knife mills, attrition mills, roll crushers, and the like are used in the first stage to provide a coarse but narrower particle size distribution feed for the second grinding stage. In the second stage, the final average particle size is attained. Sieving off both the coarse tail and the fine tail of the size distribution typically narrow the final particle size distribution further. The coarse tail is recycled to the grinder, and the fine tail may be used for other purposes or recycled back to the polymerization reactor.

\subsection{Surface Cross Linking}

Since small particles of hygroscopic polymer absorb water very fast, they can quickly form a thin, sticky layer of gel at the particle surface and then clump together or "gel block". The resulting inter particle adhesion because the formation of large, sticky agglomerates with low inter particle porosity and drastically slower swelling rate. One remedy is to add a solution of multivalent cations to the surface of the dry particles, forming an ionic ally cross-linked surface layer. Alternatively, the particle surface can be coated with and reacted at elevated temperature with polyols, poly epoxides, linear or cyclic dieters or polyamines, as examples, forming a covalently cross-linked surface shell. This shell of higher crosslink density is less swell able, less sticky, more rigid than the untreated polymer surface, and prevents gel-blocking. This improves the permeability of the particle bed toward liquids. A third method to produce these so-called structured particles is to first form a particle with higher cross-link density and then lower the cross-link density in the center of the particle cross-linked polymers made in the presence of oxidizing agents such as sodium or potassium chlorate have shown improvements in absorbency under load and swelling capacity after a high temperature heating step wherein a portion of the polymer chains in the particle center are cleaved through the action of the oxidizers.

\subsection{Why doesn't SAP dissolve in water?}

Cross-links between polymer chains form a three-dimensional network and prevent swelling to infinity i.e. dissolving. This is due to the elastic retraction forces of the network, and is accompanied by a decrease in entropy of the chains, as they become stiffer from their originally

coiled state. Water coiled cross linked chains hydrated chains. There is a balance now between the forces of retraction and tendency for the chains to swell to infinite dilution. The degree of cross-linking has a direct effect on the level of swelling of the polymer and strength of the

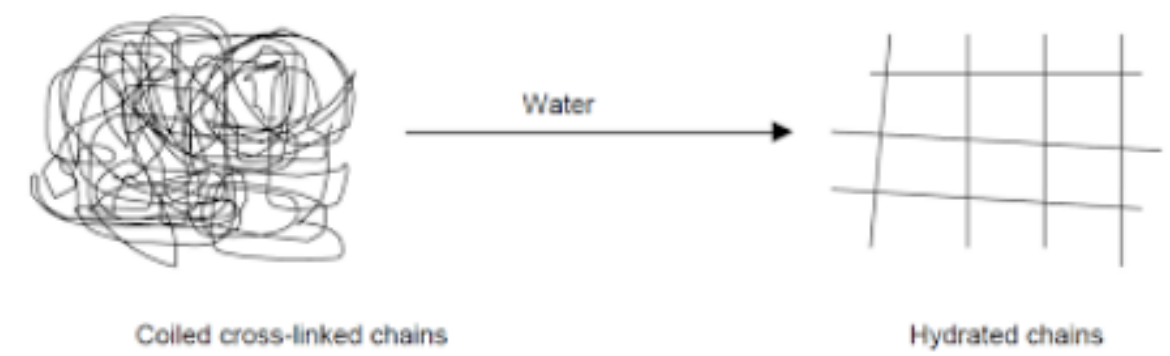

Figure4. Cross linked polymer to hydrated chain

network i.e. increased cross-link density=decreased swelling capacity=increased gel strength. Crosslinking is discussed in more detail later. Swelling in ionic polymers for ionic polymers there is another solvent/polymer interaction beyond simple mixing. The neutralized chains contain charges that repel each other. Overall electrical neutrality is maintained as the negative carboxylate groups are balanced by the positive sodium ions. Upon contact with water the sodium ions are hydrated which reduces their attraction to the carboxylate ions (due to the high dielectric constant of water). This allows the sodium ions to move freely within the network, which contributes to the osmotic pressure within the gel. The mobile positive sodium ions however, cannot leave the gel because they are still weakly attracted to the negative carboxylate ions along the polymer backbone and so behave like they are trapped by a semipermeable membrane. So the driving force for swelling is the difference between the osmotic pressure inside and outside of the gel. Increase the level of sodium outside of the gel will lower the osmotic 
pressure and reduce the swelling capacity of the gel. The maximum swelling of the gel will occur in deionized water. Body fluids such as urine contain ions including sodium, which reduce the swelling capacity of a superabsorbent compared to pure water. In order to more closely simulate the real life application of SAP i.e. in a diaper, super absorbents are normally tested in a saline solution. This simulates the effects of ion concentration on swelling capacity in application testing fluid for superabsorbent polymers.

\subsection{Analytical Evaluation}

This section contain the SAP testing method that are very useful in practical point of view for academic and industrial analysts.

\subsubsection{Swelling Absorbency Capacity}

Generally, when the term swelling or absorbency are used without specifying its contains; it implies uptake of distilled water while the sample is freely swollen, i.e., no load is put on the testing sample. There are several simple methods for the free-absorbency testing which are dependent mainly on the amount of the available sample, the sample absorbency level, and the methods precision and accuracy.

There are 3 methods to test the absorbency

- Tea bag method

- Centrifuge method

- Sieve method

\subsubsection{Tea Bag Method}

This method is the most conventional, fast, and suitable for limited amount of sample $\left(\mathrm{W}_{0}=0.1-0.3\right)$. The SAP sample is placed into the tea bag and the bag is dipped in an excess amount of water or saline solution for one hour to reach the equilibrium swelling. Then excess solution is removed by hanging the bag until no liquid is dropped off. The tea bag is weighed (W1) and the swelling capacity is calculated by equation (1). This method's precision has been determined to be around $\pm 3.5 \%$.

$\mathrm{S}_{\mathrm{e}}=\frac{(W 1-W 0)}{W 0}$

\subsubsection{Centrifuge Method}

The centrifuge method are more accurate than the tea-bag method. Thus, $0.2 \mathrm{~g}\left(\mathrm{~W}_{1}\right)$ of SAP is placed into a bag $(60 * 60 \mathrm{~mm})$ made of non-woven fabric. The bag is dipped in $100 \mathrm{~mL}$ of saline solution for half an hour at room temperature. It is taken out, and then excess solution is removed with a centrifuge separator. Then, weight of bag $\left(\mathrm{W}_{2}\right)$ is measured. The same stage are carried out with an empty bag, and the weight of bag $\left(\mathrm{W}_{0}\right)$ is measured. The swelling capacity is calculated by equation 2

$$
\mathrm{S}_{\mathrm{e}}=\frac{(W 2-W 0-W 1)}{w 1}
$$

Since the inner particle liquid is noticeably removed by this method, the measured values are often more accurate and lower than those obtained from the tea bag method.

\subsubsection{Sieve Method}

SAP sample $(\mathrm{W} 1, \mathrm{~g})$ is poured into excess amount of water or a solution and dispersed with mild magnetic stirring to each equilibrium swelling. The swollen sample is filtered at desired time through weighed 100-mesh wire gauze sieve. Then it is dewatered carefully and rapidly using a piece of soft open- cell polyurethane foam until the no longer slips from the sieve when it's held vertical. The quantities figs can be calculated by the given equation (3).

\section{DISCUSSION}

\subsection{Absorbency Under Load (AUL)}

The AUL data is usually given in the patent literature and technical data sheets by industrial SAP manufactures. When the term AUL is used without specifying its swelling media; it implies an uptake of $0.9 \%$ Nalco solution. While the testing sample is pressurized by some load. A typical AUL tester is a simple but finely made device including a macro-porous sintered glass filter plate. The weighed dried 
SAP sample is uniformly placed on the surface of polyester gauze located on the sintered glass. A cylindrical solid load is put on the dry SAP particles while it can be freely slipped in a glass cylinder. Desired load is placed on the SAP sample. Saline solution $0.9 \% \mathrm{NaCl}$ is then added when the liquid level is equal the height of the sintered glass filter. The whole set is covered to prevent surface evaporation and probable change in the saline concentration. After 60min, the swollen particle are weighed again, and AUL is calculated the following equation.

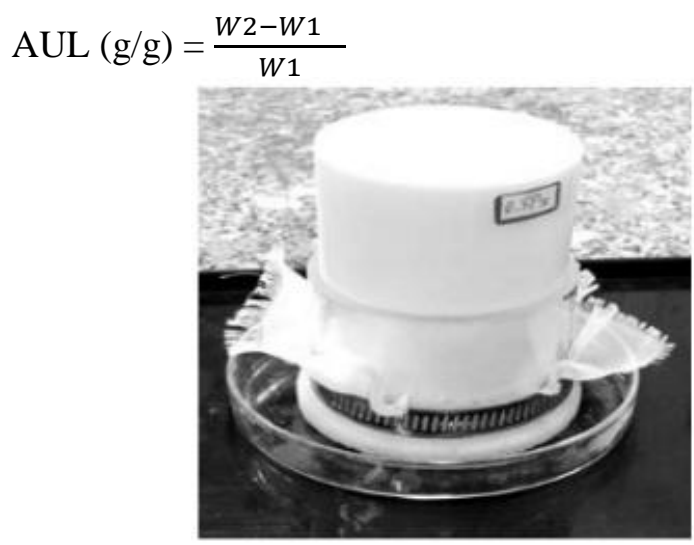

(a)

Figure5. A typical AUL tester picture (a) and various parts (b)

Where, W1 and W2 denote the weight of dry and swollen SAP, respectively. The AUL is taken as a measure of the swollen gel strength of SAP material.[7]

\subsection{Elastic Modulus}

The elastic shear modulus of swollen superabsorbent polymer is commonly measured by means of oscillatory rheometry, using any of a variety of commercially available instruments. The sample to be analyzed is screened to the desired particle size cut that the swollen particle size is smaller than the gap between the measuring plates. The sample is swollen in the desired liquid, usually $0.9 \% \mathrm{NaCL}$ solution or synthetic urine. Excess liquid is removed from the swollen hydrogel by blotting to minimize inter particle slippage. The hydrogel typically is placed onto the lower circular plate. An upper conical plate is positioned at the proper sample gap, which is packed with the gel. An oscillating torque is applied at a known frequency to the upper plate and the resulting angular displacement of the cone is determined. The shear modulus of the hydrogel is calculated from the ratio of the applied stress to the resulting strain.

Wicking capacity rate: - An originating simple test has been suggested by pioneering researchers Fanta and Doane to calculate the wicking capacity (WC) of SAP material with conventional physical appearance i.e., sugar-like particle.

Thus, SAP sample is added to a folded filter paper cone prepared from an accurately tarred circle. The cone was lightly tapped to settle the sample into the tip, and the tip of the cone is then held for $60 \mathrm{~s}$ in a $9 \mathrm{~cm}$ Petri dish containing $25 \mathrm{~mL}$ water. Water wicks up the entire length of the paper in a min. excess water is then allowed to drain from the paper by containing the tip for 60s with the circle of dry filter paper on square of absorbent towel. The weight of wet paper plus swollen polymer is determine (A), and the absorbency of the sample in $\mathrm{g} / \mathrm{g}$ is then calculated after correcting for the weight of dry paper and the amount of water absorbed under identical conditions by the paper alone in the absence of sample by equation (5). Each test is preferred to be repeated 3-5 times.

$\mathrm{WC} \frac{=(A-B-W 1)}{W 1}$

Where $\mathrm{B}$ is the wet paper without polymer

Bulk density and flow ability: - the polymer bulk density and polymer flow ability affect conveying pipe and hopper design in the polymer manufacturing plants and dosing machine settings on diaper manufacturing lines. Flow ability is typically evaluated by timing flow of a $100 \mathrm{~g}$ granular sample from a funnel into a cylinder of know volume. The bulk density, taken from the same procedure, is the mass/volume ratio of polymer in the cylinder.[7] 


\subsection{Application}

The unique attributes of superabsorbent polymer make them useful in many different application. The liquid absorption and retention ability makes them useful in disposable hygienic products. They include infant diapers, feminine hygiene pads, and adult's incontinence products. Other absorbent products suitable for superabsorbent polymers are paper towels, surgical sponges, meat trays, disposable mats for outside doorways, household pet litter, bandage, and wound dressings. Because the gel can slowly release water vapor to the atmosphere, they can be used in humidity controlling products or as soil conditioners. Superabsorbent polymers can also release water-soluble substances within the network structure into surroundings solution; hence, pharmaceuticals or fertilizers may be controllably released from within the gels. The rubbery nature of the swollen gels can control the consistency of cosmetic or concrete, and yield a soft and dry feel too hot or cold packs for sore muscles. The combinations of swelling and soft rubber properties imparts sealing properties to products that may come into contact with water or aqueous solutions, such as, underground wires and cables. In the following paragraph, additional details will be given of how properties of superabsorbent polymers affect their utility in specific products.[9]

\subsection{Hygiene and Bio Related Areas}

The most volume of SAP produced all over the world is used in disposable diapers for infants and adults. SAP are all used to produce the feminine hygiene product. Therefore, most of the research works have been focused on hygienic grades which are usually used with fluff in diapers. [6]

\subsection{Disposable Infant Diapers}

A basic disposable diaper consists of an absorbent core sandwiched between a liquid permeable top sheet and an impermeable back-sheet. The top sheet, next to the baby's skin, allows the urine to flow through it into the core. The back sheet, made of permeable plastic, helps keep the baby's clothing dry. The core takes in the liquid, distribute it within the core and holds the liquid under compression from the baby. In a diaper, the polymer is mixed with 0.5-10 time its mass of cellulose pulp fluff to make up the core. Cores containing superabsorbent are thinner because smaller volume of dry superabsorbent are thinner because a smaller volume of dry superabsorbent polymer can absorb the same volume of aqueous liquid as a larger volume of fluff. In addition to the specific absorbency of the superabsorbent polymer, the absorption rate of the diaper must be optimized to the urination rate of the baby. When the core absorbs too slowly relative to the urination rate, the liquid overflow the core and leaks from the diaper.

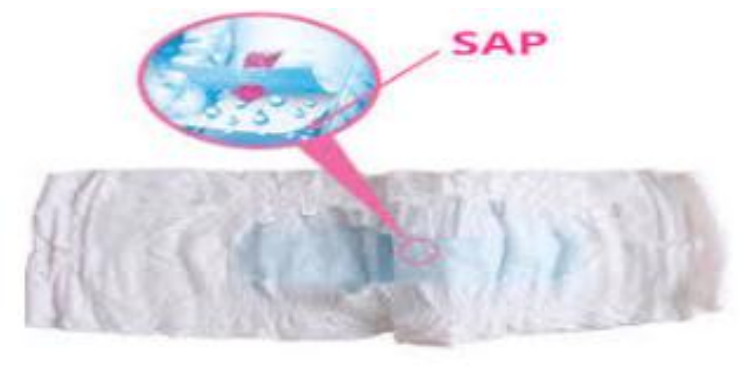

Figure6. SAP used in Diapers

The absorption rate of the composite is influenced by the absorption rate of the superabsorbent fast swelling of the polymer can contain the liquid quickly. However, in some diaper design, fast swelling may cause the diaper to leak if the porosity and permeability of the composite is reduced too rapidly.

\subsection{Adult Incontinence}

Adults Incontinence products included belted or fitted disposable garments and light incontinence pads, which are similar to feminine napkins. Similar design considerations are used as in baby diapers. In adults the liquid volume is larger and urination rate is faster than in infants; therefore, polymers with larger specific absorbency and faster swelling rate are desired for this application. The market for these products is growing with thea ging population.[14]

\subsection{Feminine Hygiene}

The first personal-hygiene product commercialized with superabsorbent polymer was a feminine napkin in japan. Perhaps the biggest challenge in using superabsorbent polymer in these products is the huge 
difference in properties of urine and menstrual fluid. Menstrual fluid is a complex, viscous mixture of water, salts, protein's and cells. The cells are far too large to be absorbed application into the SAP, and the protein makes the fluid very viscous, and therefore slows the diffuse in the polymer. In other blood absorption applications such as in surgery and surgical appliances, the polymers can be chemically modified to prevent undesired blood clotting. Incorporating similar additives into SAP may improve their performance in feminine hygiene products.[13]

\subsection{Agricultural Areas}

The presence of water in soil is essential to vegetation. Liquid water ensures the feeding of plants with nutritive elements, which makes it possible for the plants to obtain a better growth rate. It seems to be interesting to exploit the existing water potential by reducing the losses of water and also ensuring better living conditions for vegetation's. Taking into account the water imbibing characteristics of SAP materials, the possibilities of its applications in the agricultural field has increasingly been investigated to alleviate certain agricultural problems. SAP's have been successfully used as soil amendments in the horticulture industry to improve the physical properties of soil in view of increasing their water holding capacity and nutrient retention of sandy soils to be comparable to salty clay or loam. SAP hydrogel potentially influence soil permeability, density, structure, texture, evaporation, and infiltration rates of water through the soils. Particularly, the hydrogel reduce irrigation frequency and compaction tendency, stop erosion and water runs off, and increase the soil aeration and microbial activity. The hydrogels also acts as a controlled release system by favoring the uptake of some nutrients elements, holding them tightly, and delaying their dissolution. Consequently, the plant can still access some of the fertilizers, resulting in improved growth and performance rates.[14,11]

On other hand SAP's in agricultural can be used as retaining material in the form of seed additives, seed coatings, root dips, and for immobilizing plant growth regulator or protecting agents for controlled release. Additional interesting instance is a research recently conducted on the effect on SAP material on the characteristics of sport trough. Trough is of significant importance as an inseparable part of all kinds of green spaces. Irrigation water consumption trough is very huge, especially in the hot and dry climate due to surface evaporation and infiltration. However, in wet and soggy soils with low oxygen content, superabsorbent polymers can prolong the unfavorable soil conditions. Salt tolerant SAP may be more useful than the common poly acrylates for this applications.[5,11]

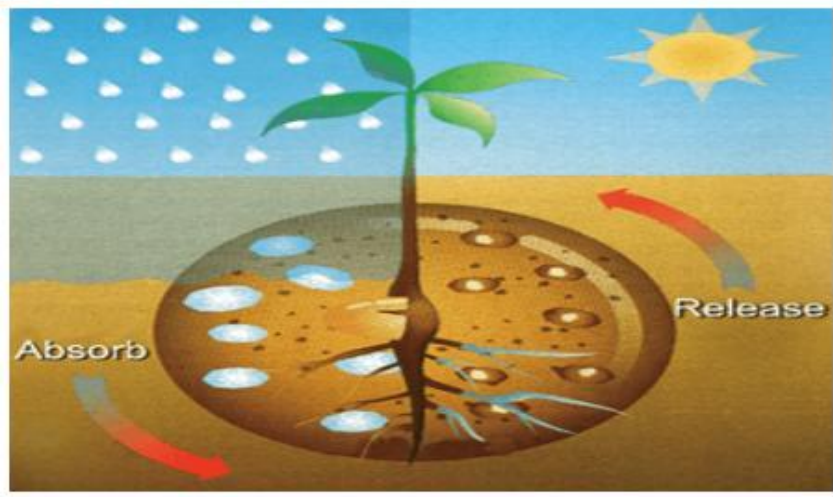

Figure7. SAP used in agricultral

\subsection{Other Areas}

In addition to the hygienic and agricultural areas, SAP material are used in many other fields, e.g., artificial snow, ornamental products, building internal decoration, fire extinguishing/retarding gels, cryogenic gels, food/meat packaging. Concrete strengthening, reduction of the ground-resistance in the electrical industry and controlled release of pesticides and agrochemicals, are other instances for the SAP.[20]

\subsection{Food Packaging}

SAP can also absorb juice or water from raw chicken, shellfish and other meats, or from frozen food as they thaw, reducing sogginess of the product. Chilled superabsorbent polymer gels can also be used as a dry-cooling medium to keep perishable food cold. The humidity controlling property of superabsorbent polymer also can be used to maintain a constant humidity in vegetable and fruit storage and prevent water spotting on fruits.[16] 


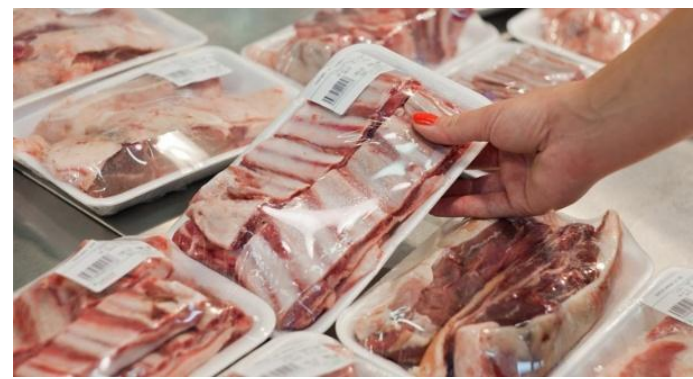

Figure8. SAP used in food packaging

\subsection{Construction Material}

Superabsorbent polymers are used to control liquid water in a variety of construction-related products. Joint-sealing composites are made by blending super absorbent into chloroprene rubber or into poly (ethylene-co-vinyl acetate). These composites are used like motor in the concrete block walls of structure. Gaps lefts during construction are subquently filled as the superabsorbent swells in any water, and subsequent leaked are prevented. A water-blocking construction backfill has also been developed from cement, water absorbing polymer, and an asphalt emulsion. The swelling property of superabsorbent also protects communication cables from water damage.[12] Water blocking tapes are wrapped around fiber optic communication cables and power transmission cables to stop in trussion of water into the cables if the water-resistant covering are cut or broken swelling polymer seals the damaged area and slow water penetration further along the cable.[10]

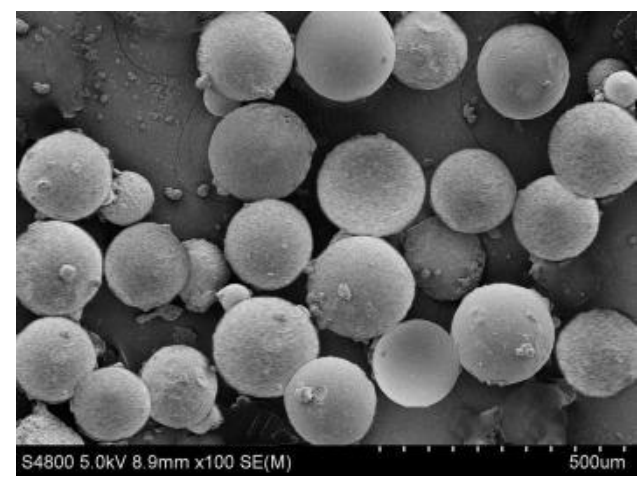

Figure9. SAP used in construction material

\subsection{Sensors}

SAP can be used in sensor system by virtue of their swell ability and rubbery nature, which are controllable by changes in water content, $\mathrm{pH}$, and ionic strength. Because a small voltage may be induced in a soft hydrogel by applying mechanical stress to the gel, a pressure-sensitive switch is possible. The potential that develops between the stressed and unstressed parts of the gel generates a signal that can light a photo diode intensity of the light emitted by the diode depends on the magnitude of the applied stress. The application of a voltage across a polyelectrolyte gel conversely causes a volume change in the gel. This Amy be used to perform work in applications such as robotic fingers or artificial muscles.[9]

\subsection{Economic Aspects}

Cross-linked, partially neutralized neutralized poly (sodium acrylate) represent the overwhelming majority of the super absorbent manufactured in the world today. The global manufacturing capacity for these SAP in 2001 was estimated to be about 1.187 million metric ton per year. This quantity is split with $380 \mathrm{kT} /$ year Europe, 427kt/year in the United States of America, and 380kt/year in Asia. Total global usage of SAP polymer in 2001 amounted to about $1093 \mathrm{kT}$, or just over $92 \%$ of the global manufacturing capability. In 2001, there were six principal global manufactures of SAP. BASF Aktiengesellschaft had about 350kt/year of manufacturing capacity, followed by Degussa with about 245 kT/year, Nippon Shokubai KagakuKogyo with about 230 kT/year, The Dow Chemical Co. with about 120kT/year, SanDia (Sanyo and Mitsubishi joint venture) with about 95kT/year, and Sumitomo Chemical with about $60 \mathrm{kT} /$ year. Several more small manufacture together made up another 132 kT/year manufacturing capability. Included in this group of smaller companies are those who make 
slightly different superabsorbent polymer such as cross-linked, s lightly ionized polyacrylamide which are used mainly in the agriculture and horticultural industries. SAP are mainly used in disposable, personal care hygiene products. About $81 \%$ of total tonnage is used in infant diapers and child training pants. Another $14 \%$ of the total is used in adult incontinence products, and about $2 \%$ is used in feminine menstrual pads and napkins. The remaining 3\% of total tonnage is used in other application such as construction materials, cable wrapping tapes, agriculture, and horticulture.

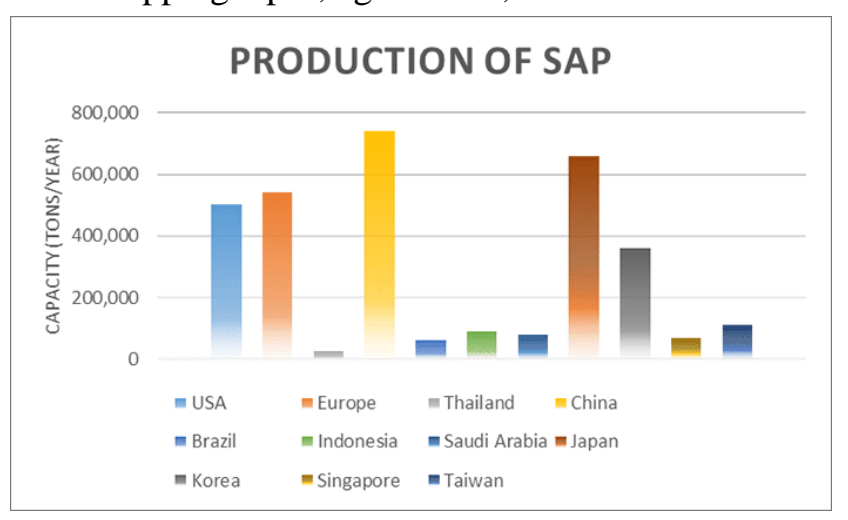

Figure10. World SAP producers

SAP material cannot return to their starting monomers, i.e., they are scientifically irreversible to toxic initiating materials. Here, like so many polymers the starting toxic is converted chemically to totally non-toxic product via polymerization reaction. SAPs are organic, materials with well-known general structure. For instance, the agriculture SAP with the same name "cross-linked acrylamide/potassium acrylate copolymer" has been recorded in the most valid data Centre of chemicals, i.e., the Chemical Abstract. In the material safety data sheet (MSDS) of the superabsorbent manufactures, they are called as "Safe and Non-toxic Material". The conventional SAP material are neutral and inert. They are moderately bio-degraded in the soil by the ionic and microbial media to convert finally to water, carbon dioxide and organic matter. Therefore, SAPs do not contaminate the soil and environment. They do not exhibit systemic toxicity. In addition, their safety in the soil has been approved by the Agriculture Ministry of France. Research has shown little or no consistent adverse effect on soil microbial populations. The environmental fate of SAPs, and their microbial degradation was investigated by many researchers. The researchers found that no toxic species were remained in soil after several-years SAP consuming.

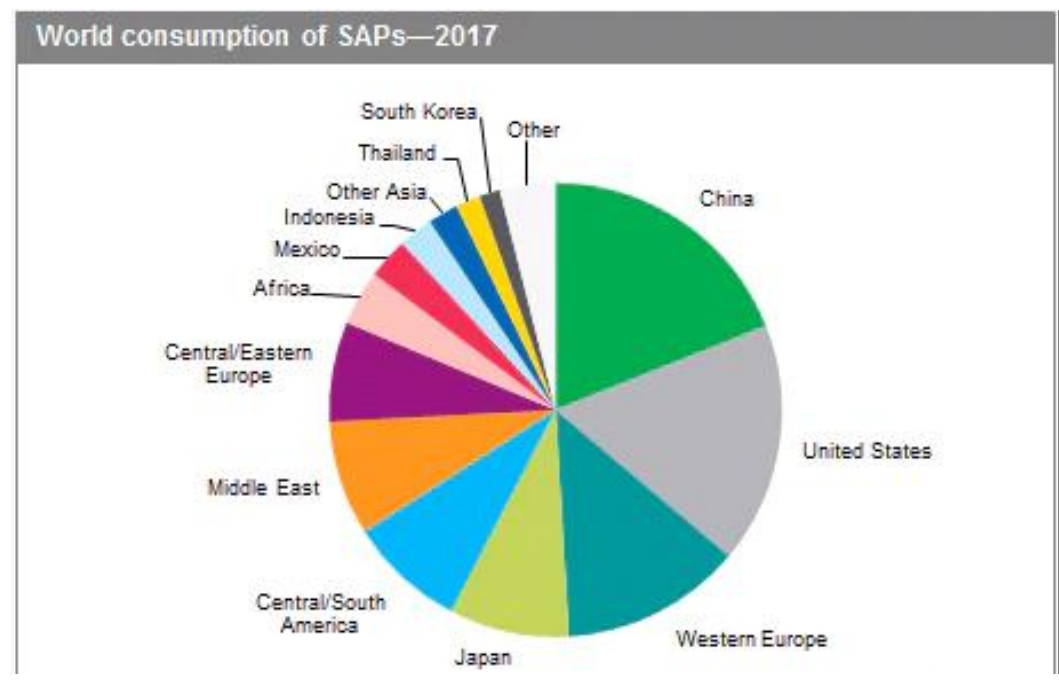

Figure11. World SAP consumer

\section{CONCLUSION}

During more than one decade research on SAP materials, we have realized that everybody is impressed by observing the surprising behavior of swelling of SAP particles poured in a glass of water. It is really fantastic however, beyond the "glass of water presentation", SAP have been applicable increasingly in many uses ranged from personal care product to agricultural. The biopolymer-contained SAPs , however, possess typically higher cost and less performance than their fully synthetic counterparts. 
Beside various application, the most volume of SAP world production is yet consumed in hygienic uses, i.e., and disposable diapers. SAPs have created a very attractive area in the viewpoint of super-swelling behavior chemistry, and designing the variety of final applications. When working in this field, we always deal with water, aqueous media and bio-related systems. Thus, we increasingly walk in a green area becoming greener via replacing the synthetics with the bio-based material, e.g., polysaccharides and polypeptides. This, however, is a long term perspective. More or less, the acrylic kingdom will extend its domination in the future markets. In spite of the SAP attractiveness, there are some drawbacks seeming to the worth nothing. Finally considering high-cost and increasing prices of crude oil, the necessity of preparing natural based SAPs seem more obvious. This gave the way to further developments in this areas in the mid and far future ahead .

\section{REFERENCES}

[1] Superabsorbent Super-Hydro-Grow made by Super Absorbent Co., www.superabsorbent. com,

[2] Stahl JD, Cameron MD, Haselbach J, Aust SD, Biodegradation of superabsorbent polymers in soil,

[3] F. L. Buchholz and N. A. Peppas, eds., Superabsorbent Polymers, Science and Technology (ACS Symposium Series 574), American Chemical Society, Washington, D.C., 1994.

[4] F. L. Buchholz and A. T. Graham, eds., Modern Superabsorbent Polymer Technology, Wiley-VCH, New York, 1998.

[5] J. P. Cohen Addad, Physical Properties of Polymeric Gels, John Wiley \& Sons, Inc., New York, 1996.

[6] D. DE Rossi, K. Kajiwara, Y. Osada , and A. Yamauchi, eds., Polymer Gels, Fundamentals and Biomedical Applications, Plenum Press, New York, 1991. • L. Brannon-Peppas and R. Harland, Absorbent Polymer Technology, Elsevier, New York, 1990.

[7] P. K. Chatterjee, ed., Absorbency, Elsevier, New York, 1985.

[8] Jensen, M, "Use of superabsorbent Polymer",Vol 35, No. 1, January 1, 2013, pages 48-52.

[9] Ekebafe, L. O., Ogbeifun, D.E., and Okieimen, F.E. "Polymer Applications ”, Biokemistri, Vol 23, No. 2, June 30, 2011, pages 81-8.

[10] Al-Nasra, Moayyad, and Daoud, Mohammad, "Investigating the use of Super Absorbent Polymer in plain concrete", IJETAE, Vol 3, Issue No. 8, August 2013, pages 598-603.

[11] Ekebafe, L. O., Ogbeifun, D.E., and Okieimen, F.E. "Polymer Applications in Agriculture", Biokemistri, Vol 23, No. 2, June 30, 2011, pages 81

[12] Mudiyanselage TK, Neckers DC, Highly absorbing superabsorbent polymer, J Polym Sci A Polym Chem,

[13] Brannon-Peppas L, Harland RS, Absorbent Polymer Technology, Elsevier, Amsterdam, Ch 1- 4, 1990.

[14] Andrade JD, Hydrogels for medical and related applications, ACS Symp. Series.

[15] Mudiyanselage TK, Neckers DC, superabsorbent polymers, 2008.

[16] Mudiyanselage TK, Neckers DC, Highly absorbing superabsorbent polymer, J Polym Sci A Polym Chem, 46, 1357-1364, 2008.

[17] Cordani P, Method of modifying weather, U.S. Patents 6,315,213, 2001.

[18] Superabsorbent Super-Hydro-Grow made by Super Absorbent Co., www.superabsorbent. com, available in 5 October 2005.

[19] Horta-Sorb Superabsorbents, Website of Horticultural Alliance, Inc., www.hortsorb.com, available in 7 April 2003.

[20] Kabiri K, Zohuriaan-Mehr MJ, Superabsorbent hydrogels from concentrated solution polymerization, Iran Polym J, 423-430, 2004.

[21] Omidian, H; Hashemi, SA, Sammes PG, Meldrum I, Modified acrylic-based superabsorbent poly- mers. Effect of temperature and initiator concentration, Polymer, 1998.

Citation: Pradnya Ingle, et.al, "Super-Absorbent Polymer: A Review on the Characteristics and Application", International Journal of Advanced Research in Chemical Science, 7(5), pp. 8-21. DOI: https:// doi.org/10.20431/2349-0403.0705002

Copyright: $\odot 2020$ Authors, This is an open-access article distributed under the terms of the Creative Commons Attribution License, which permits unrestricted use, distribution, and reproduction in any medium, provided the original author and source are credited. 